\title{
Information and Announcements
}

\section{Science Academies' Refresher Course on Quantum Mechanics and Nuclear Physics}

\author{
15-30 April 2016 \\ at Dayanand Science College, Latur
}

Sponsored by Indian Academy of Sciences, Bengaluru, Indian National Science Academy, New Delhi, The National Academy of Sciences, India, Allahabad.

Applications are invited from undergraduate college teachers. This Refresher Course aimed at helping teachers to add value to their teaching by solving problems and having tutorial work.

\footnotetext{
Tentative List of Topics -

Module 1 :- Nuclear Systematic :- Nuclear binding energy, Nuclear stability $\alpha, \beta, \gamma$ decays, Nuclear reactions, fission and fusion.

Module 2 :- Quantum Physics :- Uncertainty Principle :- Heisenberg Microscope, Time energy uncertainty wave particles to incorporate uncertainty principle using de-Broglie relation.

Module 3 :- Schrodinger Wave Equation :- Heuristic derivation, Operators associated with momentum and energy, Time independent schrodinger equation, elementary notions of operators Eigen functions and Eigen values.

Module 4 :- One dimensional problems :- particle in box, Particle in a square well potential scattering in a barrier potential, linear harmonic oscillator, expectation values of $\mathrm{X}, \mathrm{P}$ in the ground state oscillator.

Module 5 :- Hydrogen atom Problem :- Schrodinger equation bound state solution in Coulomb potential, concept of degeneracy, angular momentum Eigne function and Eigen values, The fundamental commutator between position and momentum atom in spherical polar coordinates, separation of variables.

Resource Persons :- Dr. S. H. Patil (IIT Mumbai), Dr. Kailas (Center for Advanced Technology, Indore), Dr. Avinash Khare (Phule University, Pune), Dr. A. L. Chaoudhari (S.R.T.M.University, Nanded), Dr. Rajaram Nitayananda (Azim Premji University, Banglore), Dr. Atul Mody, (Vivekanand College, Mumabi).
}

Course Director: Dr Sulbha Kulkarni, IISER, Pune

Course Coordinator : R.H. Ladda, Ex-Principal, Latur

Teachers who wish to participate should send their applications online using the following link: http://web-japps.ias.ac.in:8080/Refreshcourse/RCNP.jsp and college dsclatur.org.in. Alternatively applications may be sent with necessary personal details by email (hladda@gmail.com). Please note that selected participants will be paid III A/c fare by the shortest route with local hospitality.

Last date for receiving applications 20 January 2016. 